\title{
Profile of Patients with Breast Cancer Brain Metastasis in Abidjan
}

\author{
Kouame Konan Yvon Kouassi*, Moctar Touré, Evrard Narcisse Seka, Oumou Kimso, \\ Mudasiru Akanji Oseni, Bitti Adde Odo, Arsène Gaetan Kagambega, Innocent Adoubi \\ Oncology Department of the Treichville University Hospital, Department of Medicine and Medical Specialties, Felix \\ Houphouet-Boigny University, Abidjan, Côte d'Ivoire \\ Email: *yvonkouassi@gmail.com
}

How to cite this paper: Kouassi, K.K.Y., Touré, M., Seka, E.N., Kimso, O., Oseni, M.A., Odo, B.A., Kagambega, A.G. and Adoubi, I. (2018) Profile of Patients with Breast Cancer Brain Metastasis in Abidjan. Advances in Breast Cancer Research, 7, 201-210.

https://doi.org/10.4236/abcr.2018.72012

Received: March 17, 2018

Accepted: April 27, 2018

Published: April 30, 2018

Copyright ( 2018 by authors and Scientific Research Publishing Inc. This work is licensed under the Creative Commons Attribution International License (CC BY 4.0).

http://creativecommons.org/licenses/by/4.0/ (c) (i) Open Access

\begin{abstract}
Patients with breast cancer will develop brain metastases in $15 \%-25 \%$ of cases. Objective: To identify the profile of patients with breast cancer brain metastasis. Method: Retrospective study over 4 years in the oncology department of Treichville Hospital. All patients with breast carcinoma who had a brain metastasis during their cancer management were included. Results: 41 cases of breast carcinoma brain metastasis were collected. Patients were older than 40 years in $78 \%$ of cases. Family history of breast cancer was found in $7 \%$ of patients. More than $80 \%$ of patients had consulted with T3 and T4 tumor masses, with $100 \%$ of cases of node involvement. The brain was the second site of metastasis. The tumors were Triple negative in $48 \%$, Her2 positive in $36 \%$ and luminal in $16 \%$. Brain metastases were of late onset in the majority of cases $(68 \%)$. They were clinically characterized by headaches $(100 \%)$, most often included in an intracranial hypertension syndrome (52\%). They were multiple (60\%). Therapeutically, no surgery was performed on the metastases. Three patients received whole brain radiation therapy. All patients received systemic therapy. In terms of response, 30 cases of progression were observed after treatment and 2 cases of complete response were obtained. Survival from onset of brain metastasis was of $75 \%$ at 3 months and $2 \%$ at 12 months. Patients with longer survival rates were over 40 years old with triple negative breast cancer. Conclusion: Brain metastasis from breast cancer most often occurs in a particular clinical and pathological context in our countries.
\end{abstract}

\section{Keywords}

Breast Cancer, Brain Metastasis, Abidjan

\section{Introduction}

Breast cancer is the second leading cancer that gives brain metastasis (BM), after 
lung cancer [1]. In the diagnosis stage of localized breast cancer, the risk of developing $\mathrm{BM}$ is of $5 \%$ [2]. In the metastatic stage, this incidence increases from $6 \%$ to $16 \%$ according to some studies [3] [4].

With the improvement of systemic treatments, more and more patients with breast cancer will be affected by BMs, whose management remains a key issue for clinicians. The neurological manifestations secondary to the onset of BM are feared by patients as much as clinicians.

As the blood-brain barrier is an obstacle to the effectiveness of systemic treatments, local treatments play a key role in the therapeutic strategy. In fact, whole brain radiation therapy remains a major treatment for cerebral metastases, especially in cases of multiple lesions [3]. On the other hand, in the case of single or few brain metastases, management is more complex, and necessitates localized treatments, such as surgery or stereotaxic radiotherapy [3].

No studies have yet been conducted on breast cancer brain metastases in Côte d'Ivoire.

The objective of this study was to determine the profile of patients with metastatic cerebral breast cancer in Abidjan and their treatment modalities.

\section{Materials and Method}

Our study was conducted at the cancer ward of the Treichville University Hospital in Abidjan. It was a cross-sectional study that was both retroactive and prospective, with a descriptive and non-comparative aim, which lasted for a period of 4 years (June 2013-June 2017). It focused on patients with histologically confirmed and metastatic breast cancer. Were Included in this study all patient files which had socio-demographic, clinical, therapeutic and evolutionary data.

The histological diagnosis and the different immunohistochemical statuses of breast cancers were carried out respectively at the pathology departments of the Treichville and Cocody University hospitals and at the Plateau Central Laboratory. They were performed on micro biopsies, surgical biopsies, lumpectomies and mastectomies. The diagnosis of metastasis was done from data taken from the clinical examination, imaging reports (CT, MRI...) with or without X-rays.

Data collection was possible using a survey sheet. The data collected on this survey sheet concerned information relating to:

- The patients' (age, weight, height, history of alcohol abuse, history of hypertension or diabetes and family history of breast cancer or BRCA mutation);

- The breast cancer (date of diagnosis, initial tumor size, lymph node involvement and site, initial metastatic locations and number, TNM stage, histological type, SBR modified by Elston and Ellis stage, Her2 and Hormonal status, Ki-67);

- The cerebral metastasis (the date of diagnosis, the chronology and time of occurrence, the circumstances of onset, the methods of diagnosis, the site and number, the treatments received and the therapeutic response obtained);

- The outcome (overall survival, survival since metastasis, date of last contact). 
Data processing and analysis was made possible by IBM SPSS Statistics Version 21.0 and Microsoft Excel 2013 software.

\section{Results}

During this study period, 41 cases of breast cancer brain metastasis were collected. The average age of patients was 43 with extremes of 27 and 68 years. In $78 \%$ of cases, patients were over 40 years of age. Family history of breast cancer was found only in $7 \%$ of patients (Table 1).

More than $80 \%$ of patients were first seen with bulky T3 and T4 masses, with lymph node involvement in $100 \%$ of cases. Locally advanced and metastatic stages were found in $96 \%$ of cases. The brain was the second site of metastasis. Immunohistochemically, $48 \%$ Triple negative, $36 \%$ Her 2 positive and $16 \%$ luminal (Table 2) were noted.

Cerebral metastasis onset was late in the vast majority of cases $(68 \%)$. They occurred in $92 \%$ of cases after 6 months. They were clinically characterized by headaches in $100 \%$ of cases, most often included in an intracranial hypertension syndrome (52\%). Computed tomography was the most used imaging test in our series (93\%) (Image 1). In 60\% of cases, these were multiple secondary cerebral locations (Table 3 ).

In terms of treatment, no patient had metastasis surgery. Only 3 patients received whole brain radiotherapy. The vast majority of patients received systemic

Table 1. Socio-demographic characteristics.

\begin{tabular}{|c|c|c|}
\hline Parameters & Number & Percentage (\%) \\
\hline \multicolumn{3}{|l|}{ Age } \\
\hline$\leq 40$ years & 13 & 32 \\
\hline$>40$ years & 28 & 78 \\
\hline \multicolumn{3}{|l|}{ Gender } \\
\hline Female & 41 & 100 \\
\hline Male & 00 & 00 \\
\hline \multicolumn{3}{|c|}{ High blood pressure } \\
\hline Yes & 03 & 08 \\
\hline No & 38 & 92 \\
\hline \multicolumn{3}{|l|}{ Diabetes } \\
\hline Yes & 05 & 12 \\
\hline No & 36 & 88 \\
\hline \multicolumn{3}{|l|}{ Alcohol Abuse } \\
\hline Yes & 16 & 40 \\
\hline No & 25 & 60 \\
\hline \multicolumn{3}{|l|}{ Tobacco Abuse } \\
\hline Yes & 03 & 08 \\
\hline No & 38 & 92 \\
\hline \multicolumn{3}{|c|}{ Family history of cancer } \\
\hline Yes & 03 & 07 \\
\hline No & 38 & 93 \\
\hline
\end{tabular}

The average age of patients was 43 with extremes of 27 and 68 years. In $78 \%$ of cases, patients were over 40 years of age. Family history of breast cancer was found only in $7 \%$ of patients. 
Table 2. Breast cancer clinical and pathological characteristics.

\begin{tabular}{|c|c|c|}
\hline Parameters & Number & Percentage (\%) \\
\hline \multicolumn{3}{|l|}{ Tumor initial size } \\
\hline $\mathrm{T} 1$ & 00 & 00 \\
\hline $\mathrm{T} 2$ & 06 & 15 \\
\hline T3 & 12 & 29 \\
\hline $\mathrm{T} 4$ & 23 & 56 \\
\hline \multicolumn{3}{|l|}{ Node involvement } \\
\hline N0 & 00 & 00 \\
\hline $\mathrm{N}+$ & 41 & 100 \\
\hline \multicolumn{3}{|l|}{ Initial stage } \\
\hline I & 00 & 00 \\
\hline II & 01 & 04 \\
\hline III & 25 & 60 \\
\hline IV & 15 & 36 \\
\hline \multicolumn{3}{|l|}{ First location of metastasis } \\
\hline Bone & 10 & 24 \\
\hline Brain & 08 & 20 \\
\hline Lung & 07 & 17 \\
\hline Liver & 07 & 17 \\
\hline Skin & 05 & 13 \\
\hline \multicolumn{3}{|l|}{ Histological type } \\
\hline Infiltrating Ductal Carcinoma & 40 & 98 \\
\hline Micropapillary Carcinoma & 01 & 02 \\
\hline \multicolumn{3}{|c|}{ SBR Grade modified by Elston and Ellis } \\
\hline Grade I & 01 & 02 \\
\hline Grade II & 10 & 25 \\
\hline Grade III & 29 & 71 \\
\hline Not specified & 01 & 02 \\
\hline \multicolumn{3}{|l|}{ Immunohistochimistry Profile } \\
\hline Luminal (A et B) & 06 & 16 \\
\hline HER 2 positive & 15 & 36 \\
\hline Triple negative & 20 & 48 \\
\hline
\end{tabular}

More than $80 \%$ of patients were first seen with bulky T3 and T4 masses, with lymph node involvement in $100 \%$ of cases. Locally advanced and metastatic stages were found in $96 \%$ of cases. The brain was the second site of metastasis. Immunohistochemically, $48 \%$ Triple negative, $36 \%$ Her 2 positive and $16 \%$ luminal were noted.

therapy, namely palliative chemotherapy $(76 \%)$ with or without hormone therapy or targeted therapy. Platinum based chemotherapy was used in $31 \%$ of cases (Table 3). The other anti-cancer molecules used, with or without platinum salts, were irinotecan (4 cases), taxanes (14 cases), anthracyclines (6 cases) and gemcitabine (4 cases).

In terms of response to treatment, 30 cases of progression were observed after treatment. 2 cases of complete response were obtained, one after whole brain radiotherapy, and the other after 4 courses of chemotherapy with Irinotecan-Cisplatin protocol (Image 2).

Overall survival was $84 \%$ at 6 months, $65 \%$ at 12 months, and $8 \%$ at 24 months (Figure 1). Survival at onset of BM was $75 \%$ at 3 months, $28 \%$ at 6 
Table 3. Brain metastasis characteristics.

\begin{tabular}{ccc}
\hline Parameters & Number & Percentage (\%) \\
\hline Chronology of onset & 08 & 20 \\
Synchronous & 05 & 12 \\
Metachronous & 28 & 68 \\
Late & & \\
Delay before onset & 03 & 08 \\
$\leq 6$ months & 38 & 92 \\
$>6$ months & & \\
Circumstances of discovery & 41 & 100 \\
Headaches & 21 & 52 \\
Intracranial hypertension & 12 & 29 \\
Memory disorder & 7 & 16 \\
Balance disorder & & \\
Brain imaging & 38 & 93 \\
CT & 3 & 07 \\
MRI & &
\end{tabular}

Number of brain metastases

\begin{tabular}{ccc}
$1-3$ & 16 & 40 \\
$>3$ & 25 & 60 \\
\hline $\begin{array}{c}\text { Treatments received } \\
\text { Symptomatic treatments (steroids, } \\
\text { anticonvulsants) }\end{array}$ & 41 & 100 \\
$\quad$ Surgery & & \\
Radiotherapy & 00 & 00 \\
$\quad$ Chemotherapy & 03 & 07 \\
Hormonal therapy & 31 & 76 \\
Chemotherapy regiment & 08 & 20 \\
With platinum salts & 08 & 20 \\
Without platinum salts & 13 & 31 \\
Targeted therapy (bevacizumab, herceptin) & 28 & 69 \\
\hline Lack of financial needs & & \\
Poor performance status & 38 & 93 \\
\hline Response to treatment & 03 & 07 \\
Complete response & & 05 \\
Partial response & 2 & 00 \\
Stabilization & 0 & 22 \\
Progression & 9 & 73 \\
\hline
\end{tabular}

Cerebral metastasis onset was late in the vast majority of cases (68\%). They occurred in $92 \%$ of cases after 6 months. They were clinically characterized by headaches in $100 \%$ of cases. Computed tomography was the most used imaging test in our series (93\%). In $60 \%$ of cases, these were multiple secondary cerebral locations. Only 3 patients received whole brain radiotherapy. The vast majority of patients received systemic therapy, namely palliative chemotherapy $(76 \%)$ with or without hormone therapy or targeted therapy. Platinum based chemotherapy was used in $31 \%$ of cases. In terms of response to treatment, 30 cases of progression were observed after treatment.

months, and $2 \%$ at 12 months (Figure 2). Patients with better survival were patients over 40 years of age with triple negative breast cancer (Figure 3 and Figure 4). 

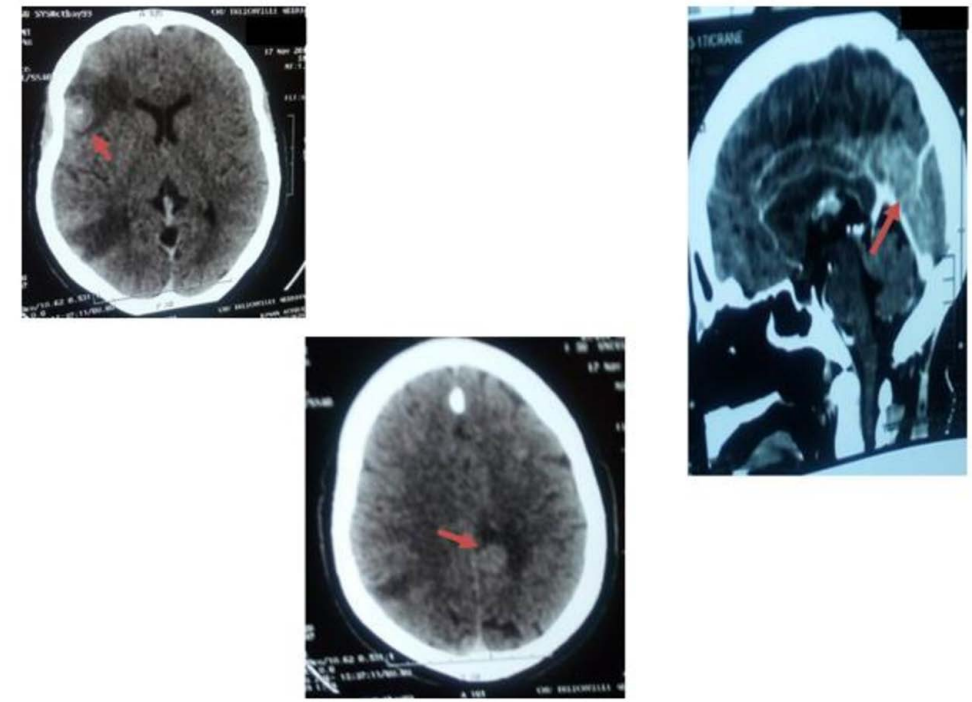

Image 1. Images of brain metastasis with significant peri-tumor edema (archive of the oncology department of Treichville University Hospital).
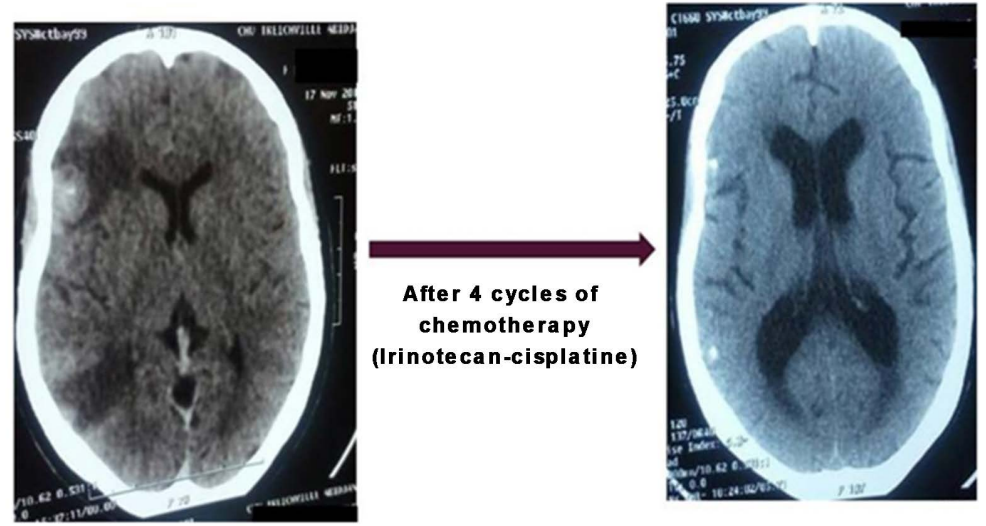

Image 2. Complete disappearance of the $\mathrm{BM}$ after 4 cycles of chemotherapy.

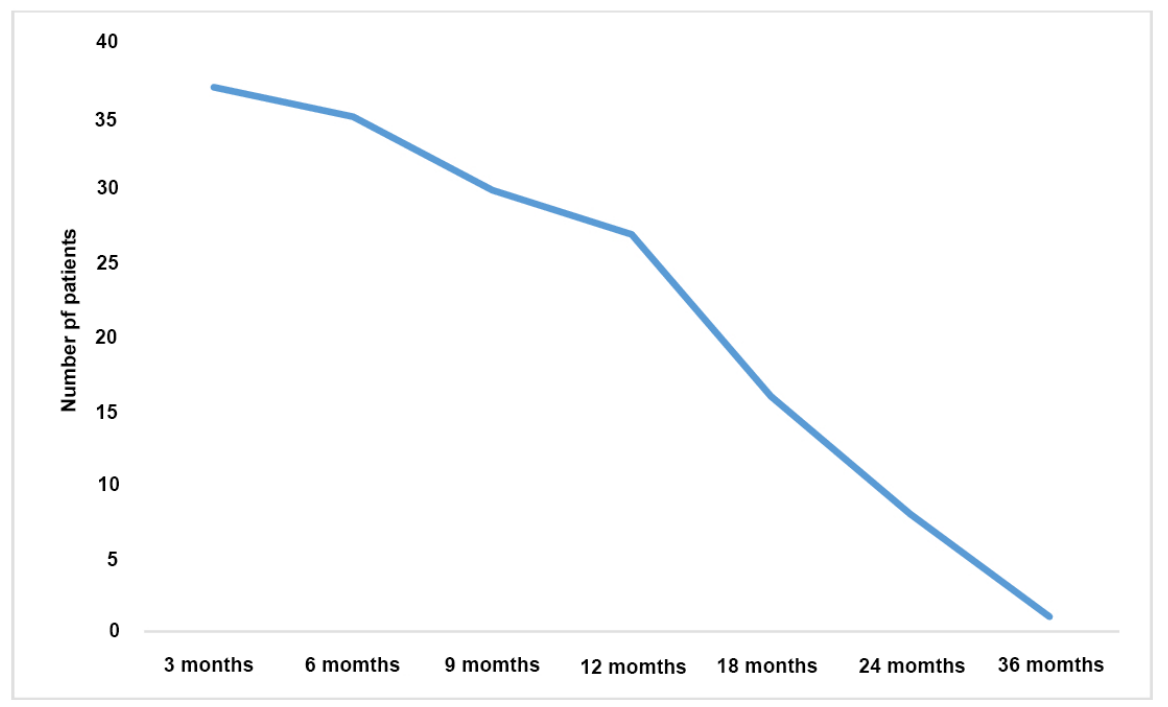

Figure 1. Overall survival. 
35

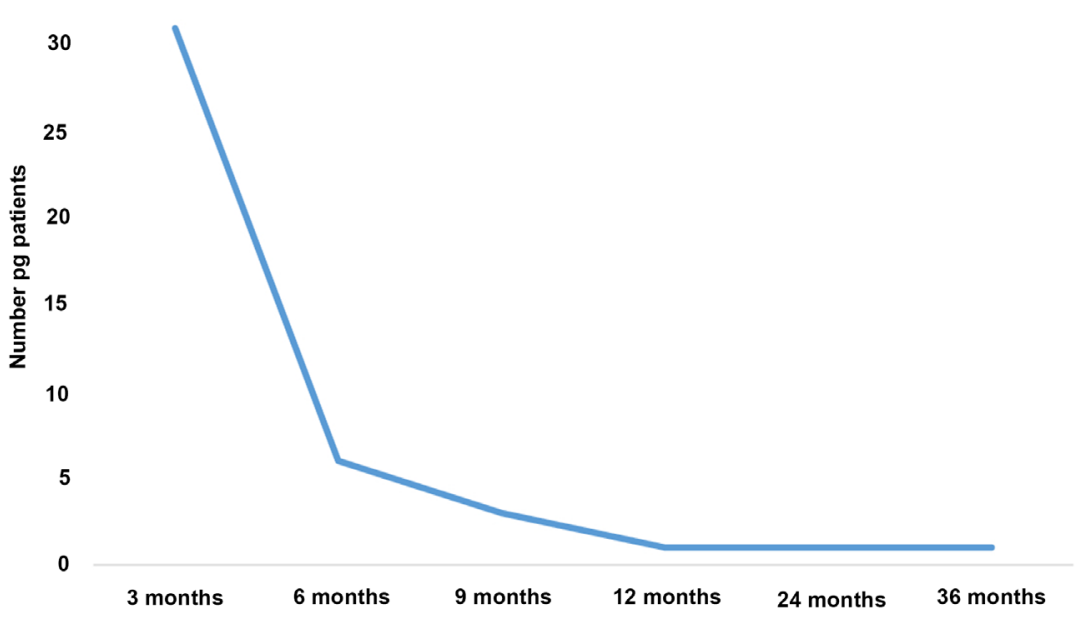

Figure 2. Survival from onset of BM.

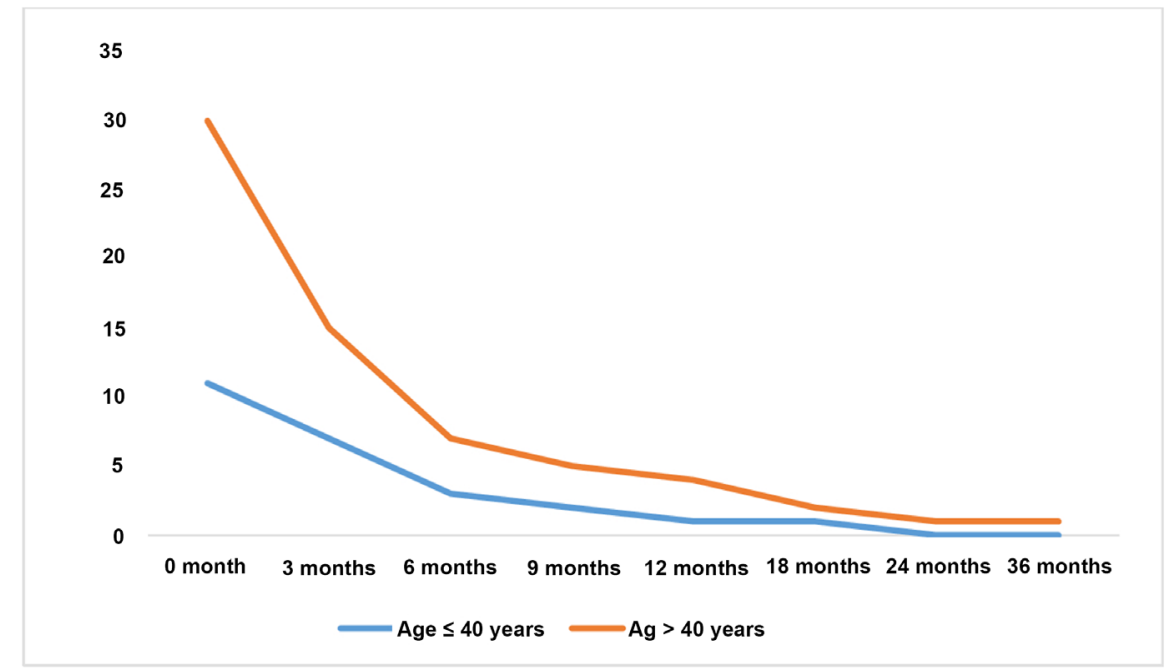

Figure 3. Survival from onset of BM and according to age.

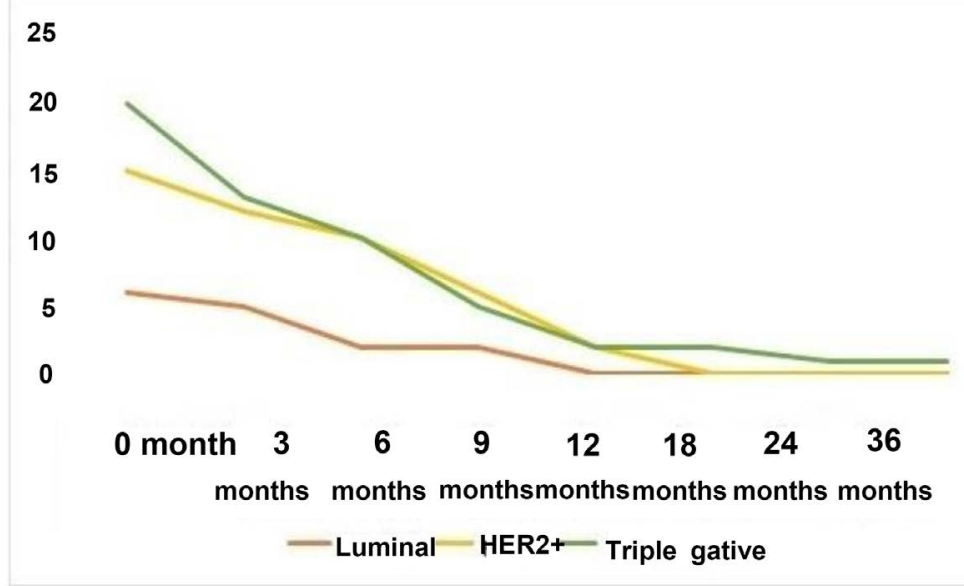

Figure 4. Survival from onset of BM and according to the immunohistochemistry staus. 


\section{Discussion}

This preliminary study carried out in the Oncology Department of the Treichville University Hospital highlights some particular characteristics of breast cancer patients who develop brain metastasis.

In terms of general characteristics, BM were more common in patients over 40 years of age. This finding is not consistent with data from Western literature that finds young age as an independent factor in the occurrence of BM. In fact, Evans [5] in 2004 found the age under 40 to be an independent predictor of BM occurrence. This low rate of BM in the under 40 population in our study can be explained not only by our small sample size, but also by the fact that adequate imaging in not always done in our context.

The onset of BM is not correlated with comorbidity factors such as high blood pressure or diabetes, nor was it with alcohol abuse. No study to date has allowed to associate smoking with the onset of BM.

In our series, family history of breast cancer (7\%) appears to be relatively high. It is accepted that the hereditary factor is of poor prognosis with the consequence of early onset of metastatic localization including cerebral. In fact, Albiges and Petit [3] [6] found that BRCA1 mutation was a risk factor for occurrence of BM.

Our results concerning tumor size, node involvement, initial stage are the same breast cancer characteristics found in other African studies [7] [8]. They are generally of poor prognostic, correlated with a high risk of secondary cerebral localization.

In our context, BM are usually of late onset. This could be explained by an adequate initial management of the mammary lesions. According to Petit [3], the median time of onset of brain metastases after breast tumor diagnosis was 22 months for triple-negative tumors, 30 months for HER2 overexpressing tumors, and 63 months for other tumor types. Overexpression of HER2 is also a risk factor for cerebral metastatic spread. According to Gabos [9], the frequency of onset of brain metastases as the first site of metastatic relapse was $0.4 \%$ for tumors without overexpression of HER2, and 9\% for tumors overexpressing HER2.

Brain metastases have the same clinical presentation, regardless of their primitive origin [10]. The clinical polymorphism is explained by the frequency of multiple metastatic sites in our series. Indeed, only $20 \%$ to $30 \%$ of $\mathrm{BM}$ are unique [2].

The diagnosis of $\mathrm{BM}$ was made by correlating imaging with the clinical symptoms. In our series, cerebral computed tomography was the most used. However, magnetic resonance imaging (MRI) has demonstrated superiority over CT scans. MRI is more efficient than CT scan in asserting unique or multiple brain metastases: $20 \%$ of patients who have a single CT lesion will probably actually have multiple lesions on the MRI [11] [12].

The therapeutic management is multimodal associating not only with specific treatment (for control of the outcome of the disease), but also nonspecific treat- 
ments for symptomatic purposes. Symptomatic treatment is essential because it aims to reduce peritumor edema and to prevent possible epileptic seizures. It consists of corticosteroid therapy, possibly associated with anticonvulsant treatment [3].

These treatments are very rarely given to our patients in an effective way because of their financial difficulties and the lack of availability of radiotherapy in our country. Indeed, radiotherapy was nonexistent in Ivory Coast.

Whole brain radiation therapy remains the reference in care for cerebral metastasis, particularly in patients with multiple metastases, or metastases that are too large to allow radiosurgery [1] [3] [10]. This radiation therapy is also indicated in patients whose disease progresses after surgery or radiosurgery of brain metastases. The objective response rate for radiotherapy is close to $60 \%$ [3] [13]. This response can be delayed several weeks after the end of the radiation [14].

Regarding chemotherapy, the choice of the protocol takes into account the immunohistochemical profile of the breast cancer. The majority of our patients being triple negative, platinum salts had been widely used in different treatment protocols.

Targeted anti-HER2 therapies were also prescribed for brain metastases of HER2 overexpressing tumors. Trastuzumab was also effective against metastasis. The median survival time after diagnosis of brain metastasis was significantly higher for HER2+ patients treated with Trastuzumab compared to HER2+ patients treated without Trastuzumab (11.6 months versus 6.1 months; 0.03). It was the same result when the comparison was done for patients with HER2tumors (11.6 months versus 6.3 months, $\mathrm{p}<0.001)$ [3].

Despite these specific therapies, tumor progression was most often observed with an accentuation of clinical symptoms and sometimes death of our patients. $\mathrm{BM}$ is the ultimate phase of cancer evolution with an impact on survival [15].

The various tumor characteristics mentioned above are responsible for the low survivals rate in our study. These survival rates differ according to the patient's status, especially the delay in metastasis (better survival for longer delays); age of the patient (better survival for elderly subjects). On the other hand, triple negative status, reputed to be of poor prognosis with a short survival rate, was not found in our series. This would most likely be due to the low sampling of our series.

\section{Conclusion}

This study serves as a plea for early management of breast cancers that are at high risk of developing metastases in our country. It is therefore important to include the management of breast cancer within the framework of multidisciplinarity.

\section{References}

[1] Lin, N.U., Bellon, J.R. and Winer, E.P. (2004) CNS Metastases in Breast Cancer. Journal of Clinical Oncology, 22, 3608-3617. 
[2] Barnholtz-Sloan, J.S., Sloan, A.E., Davis, F.G., Vigneau, F.D., Lai, P. and Sawaya, R.E. (2004) Incidence Proportions of Brain Metastases in Patients Diagnosed (1973 to 2001) in the Metropolitan Detroit Cancer Surveillance System. Journal of Clinical Oncology, 22, 2865-2872. https://doi.org/10.1200/JCO.2004.01.175

[3] Petit, T. (2010) Métastases cérébrales et cancer du sein. La Lettre du Cancérologue, XIX, 134-137. https://doi.org/10.1200/JCO.2004.12.149

[4] Pestalozzi, B.C., Zahrieh, D., Price, K.N., Holmberg, S.B., Lindtner, J. and Collins, J. (2006) Identifying Breast Cancer Patients at Risk for Central Nervous System (CNS) Metastases in Trials of the International Breast Cancer Study Group (IBCSG). Annals of Oncology, 17, 935-944. https://doi.org/10.1093/annonc/mdl064

[5] Evans, A.J., James, J.J., Cornford, E.J., Chan, S.Y., Burrell, H.C. and Pinder, S.E. (2004) Brain Metastases from Breast Cancer: Identification of a High-Risk Group. Clinical Oncology (Royal College of Radiologists (Great Britain)), 16, 345-349. https://doi.org/10.1016/j.clon.2004.03.012

[6] Albiges, L., André, F., Balleyguier, C., Gomez-Abuin, G., Chompret, A. and Delaloge, S. (2005) Spectrum of Breast Cancer Metastasis in BRCA1 Mutation Carriers: Highly Increased Incidence of Brain Metastases. Annals of Oncology off Journal European Society of Medical Oncology ESMO, 16, 1846-1847. https://doi.org/10.1093/annonc/mdi351

[7] Hsairi, M., Fakhfakh, R., Ben Abdalah, M., Jlidi, R. and Sellami, A. (2002) Assessment of Cancer Incidence in Tunisia 1993-1997. La Tunisie médicale, 80, 57-64.

[8] Touré, M., Kouassi, Y.K.K., Séka, E.N., Bambara, T.A., Yao, I., Djanhan, L.E. and Adoubi, I. (2015) Caractéristiques épidémio-cliniques des cancers du sein triple négatif à Abidjan. Carcinologie Clinique en Afrique, 14, 32-36.

[9] Gabos, Z., Sinha, R. and Hanson, J. (2006) Prognostic Significance of Human Epidermal Growth Factor Receptor Positivity for the Development of Brain Metastasis after Newly Diagnosed Breast Cancer. Journal of Clinical Oncology, 24, 5658-5663. https://doi.org/10.1200/JCO.2006.07.0250

[10] Lin, N.U., Wefel, J.S. and Lee, E.Q. (2013) Challenges Relating to Solid Tumour Brain Metastases in Clinical Trials, Part 2: Neurocognitive, Neurological, and Quality-of-Life Outcomes. A Report from the RANO Group. The Lancet Oncology, 14, e407-e416. https://doi.org/10.1016/S1470-2045(13)70308-5

[11] Schellinger, P.D., Meinck, H.M. and Thron, A. (1999) Diagnostic Accuracy of MRI Compared to CCT in Patients with Brain Metastases. Journal of Neurooncology, 44, 275-281. https://doi.org/10.1023/A:1006308808769

[12] Davis, P.C., Hudgins, P.A. and Peterman, S.B. (1991) Diagnosis of Cerebral Metastases: Double Dose Delayed CT vs. Contrast-Enhanced MR Imaging. American Journal of Neuroradiology, 12, 293-300.

[13] Ranjan, T. and Abrey, L. (2009) Current Management of Metastatic Brain Disease. Neurotherapeutics, 6, 598-603. https://doi.org/10.1016/j.nurt.2009.04.012

[14] Belkacémi, Y. and Kuten, A. (2008) Are Volumetric Changes of Brain Metastases the Best Evaluation of Efficacy? Journal of Clinical Oncology, 26, 5137-5138. https://doi.org/10.1200/JCO.2008.19.0306

[15] Pestalozzi, B. and Castiglione, M. (2008) On Behalf of the ESMO Guidelines Working Group. Primary Breast Cancer: ESMO Clinical Recommendations for Diagnosis, Treatment and Follow-Up. Annals of Oncology, 19, ii7-ii10. https://doi.org/10.1093/annonc/mdn071 